Proc Indian Natn Sci Acad 81 No. 1 February 2015 Special Issue, pp. 107-115

(C) Printed in India.

DOI: $10.16943 /$ ptinsa/2015/v81i1/48058

\title{
Effect of Flow on Heavy Quark Damping Rate in Hot QCD Plasma SREEMOYEE SARKAR ${ }^{*}$
}

\author{
Tata Institute of Fundamental Research, Homi Bhabha Road, Mumbai 400 005, India
}

(Received on 20 May 2014; Accepted on 2 September 2014)

\begin{abstract}
We determine an expression for the heavy quark damping rate in a viscous QCD plasma using kinetic theory. Shear flow in a medium changes both boson and fermion distribution functions which eventually modify heavy quark damping rate. In presence of non-zero velocity gradient in the medium all the bath particles are out of equilibrium. In this scenario we estimate the first order correction in $(\mathrm{O}(\eta / s))$ for the damping rate. The transverse domain remains infrared divergent even after hard thermal loop corrections as one encounters in case of ideal plasma.
\end{abstract}

Key Words : Heavy Quark; Shear Viscosity; Damping Rate; Infrared Divergence; HTL Resummation; Relaxation Time Approximation

\section{Introduction}

Interaction or damping rates of parton scattering processes in a thermalized QGP has been studied since past decades (Thoma and Gyulassy, 1991; Thoma, 1995; Blaizot and Iancu, 1996; Blaizot and Iancu, 1997). The interaction rate is an important quantity to study the thermalization time of a pre-equilibrium parton gas in ultrarelativistic heavy ion collisions (Thoma and Gyulassy, 1991; Thoma, 1995). The calculation is valid only for the situation which does not initiate far from equilibrium. Furthermore, scattering rates are the basic inputs for the calculation of the drag and diffusion coefficients (Bjorken, 1982 unpublished; Svetitsky, 1988; Braaten and Thoma, 1991; Moore and Teaney, 2005; Dutt-Mazumder et al., 2005). These coefficients are essential to study the particle spectra using the Fokker Planck equation in heavy ion collision experiments. Physically, interaction rate is related to the collisions with the other particles of the medium. It has been seen from the very first attempts of the calculation of damping rate $(\gamma)$ that in naive perturbation theory $\gamma$ becomes infrared divergent. However, in the non-relativistic plasma, where Coulomb interaction is the only relevant interaction, it is well known that the interaction is screened and the screening effect enters through

*Author for Correspondence : E-mail: sreemoyee.sarkar@tifr.res.in 
the effective boson propagator. With this effect taken into account $\gamma$ turns out to be finite. However, the problem becomes non-trivial while dealing with the relativistic plasma where one has to consider both the electric and the magnetic interactions. The transverse sector remains divergent even after inclusion of the plasma corrections (Blaizot and Iancu, 1996; Blaizot and Iancu, 1997; Sarkar and Dutt-Mazumder, 2013; Sarkar, 2014). This is because the latter is only dynamically screened. Finite result can be obtained using another resummation scheme developed by Bloch-Nordsieck propagator (Blaizot and Iancu, 1996; Blaizot and Iancu, 1997; Boyanovsky et al., 1998).

In recent years, the general belief is that the matter produced in Heavy Ion Collider experiments behaves like nearly ideal fluid. The success of ideal hydrodynamics explains the lower bounds on $\eta / s$ ( $\eta$ is shear viscosity and $s$ entropy density) and also both the hadron transverse momentum spectra and elliptic flow $\left(v_{2}\left(p_{T}\right)\right)$ in the low $p_{T}$ region. Above $p_{T} \gg 2 \mathrm{GeV}$, the ideal hydrodynamic description breaks down beyond which $v_{2}\left(p_{T}\right)$ does not rise as predicted by non-viscous hydrodynamics (Teaney, 2003; Dusling et al., 2010). Recently, several investigations have shown that the falling trend of $v_{2}\left(p_{T}\right)$ in the higher $p_{T}$ region can be elucidated by invoking non-ideal (viscous) hydrodynamics. It is to be noted that in viscous fluid the energy momentum tensor $\left(T^{\mu \nu}\right)$ along with the non-interacting part receives a correction term $\delta T^{\mu \nu}$ involving both the coefficients of shear $(\eta)$ and bulk viscosity $(\zeta)$.

The non-vanishing $\eta$ modifies the particle distribution function as well as equation of motion. The distribution function like stress energy tensor now will have a viscous correction term $\delta f$, i.e., for the fluid constituents and we shall write $f=f^{0}+\delta f$, where, $f^{0}$ is the local thermal distribution function (Dusling et al., 2010). In general, $\delta f$ contains both the shear and the bulk viscosity coefficients and can be obtained by solving the linearized Boltzmann equation. Here, we restrict ourselves to the modification of $\delta f$ due to $\eta$ only.

In recent years, several efforts have been made to understand the impact of these dissipative phenomena on various high energy heavy ion experimental observables like particle spectra, Handbury Brown-Twiss (HBT) radii and elliptic flow (Teaney, 2003). Recently, the impact of non-zero $\eta$ on the photon and the dilepton production rates have been addressed in (Dusling and Lin, 2008; Dusling, 2010; Bhatt et al., 2010). Attempts have also been made to calculate the drag and diffusion co-efficients and heavy quark energy loss including the viscous corrections (Das et al., 2013; Sarkar, 2014). We, here, intend to calculate the heavy quark damping rate in presence of longitudinal shear flow with viscous correction upto $\mathcal{O}(\eta / s)$. The viscous part is operative only when there exists momentum anisotropy, i.e., there exists a non-zero velocity gradient in plasma. This is a major departure from the all previous damping rate calculations where all the bath particles are assumed to be in equilibrium. 


\section{Formalism}

The motion of a heavy quark in QCD plasma looks similar to that of test particle in plasma. Thus the problem resembles to Brownian motion problem where quarks are rendering random motion and their distribution obeys Boltzmann equation. In absence of external force and gradients of temperature, velocity or density on the injected parton, the Boltzmann equation becomes,

$$
\frac{\partial f_{p}}{\partial t}=-\mathcal{C}\left[f_{p}\right]
$$

right hand side of the above equation being the collision integral.

In the current paper we are concerned with a high energy heavy quark of mass $m_{Q}$ and momentum $p$ which scatters off the light quarks $\left(m_{q} \ll g T\right)$ and gluons. The injected parton distribution has a fluctuating part $\left(f_{p}=\delta f_{p}\right)$, whereas all the bath particles are affected by the velocity gradient of the medium. Since, the injected parton is high energetic $E_{p} \gg T$ the equilibrium part of the distribution function vanishes. The collision integral for $2 \rightarrow 2$ scattering $\left(P+K \rightarrow P^{\prime}+K^{\prime}\right)$ processes then can be written as,

$$
\begin{aligned}
\mathcal{C}\left[f_{p}\right] & =\frac{1}{2 E_{p}} \int \frac{d^{3} k}{(2 \pi)^{3} 2 k} \frac{d^{3} p^{\prime}}{(2 \pi)^{3} 2 E_{p}^{\prime}} \frac{d^{3} k^{\prime}}{(2 \pi)^{3} 2 k^{\prime}} \delta f_{p} f_{k}\left(1 \pm f_{k^{\prime}}\right) \\
& \times(2 \pi)^{4} \delta^{4}\left(P+K-P^{\prime}-K^{\prime}\right) \frac{1}{2} \sum_{\text {spin }}|\mathcal{M}|^{2}
\end{aligned}
$$

The difference of the thermal phase space here with that of the light quarks in (Dutt-Mazumder et al., 2005) is an important point to note here. To arrive at the above equation for high energy partons the possibility of back scattering has been excluded and the approximation $\left(1 \pm f_{E_{p}^{\prime}}^{0}\right) \simeq 1$ has also been implemented in the thermal phase space since $E_{p^{\prime}} \gg T$. In relaxation time approximation with the help of Eqs. (1) and (2) the expression for damping rate can be expressed as shown below,

$$
\frac{\partial \delta f_{p}}{\partial t}=-\mathcal{C}\left[f_{p}\right]=-\delta f_{p} \gamma(p)^{0}
$$

where, $\gamma(p)^{0}$ can be identified as the particle interaction rate,

$$
\begin{aligned}
\gamma_{p}^{0} & =\frac{1}{2 E_{p}} \int \frac{d^{3} k}{(2 \pi)^{3} 2 E_{k}} \frac{d^{3} p^{\prime}}{(2 \pi)^{3} 2 E_{p}^{\prime}} \frac{d^{3} k^{\prime}}{(2 \pi)^{3} 2 E_{k}^{\prime}} \\
& \times\left(f_{k}+\delta f_{k}^{\eta}\right)\left(1 \pm f_{k^{\prime}} \pm \delta f_{k^{\prime}}^{\eta}\right)(2 \pi)^{4} \delta^{4}\left(P+K-P^{\prime}-K^{\prime}\right) \frac{1}{2} \sum_{\text {spin }}|\mathcal{M}|^{2} .
\end{aligned}
$$

Now, if a gradient of flow is present in the medium the distribution of bath particles gets modified. One writes viscous corrected distribution function as $f_{i}=f_{i}^{0}+\delta f_{i}^{\eta}\left(\delta f_{i}^{\eta} \ll f_{i}^{0}\right)$, where, $i=k, k^{\prime} . \delta f_{i}^{\eta}$ is the first order viscous correction to the thermal distribution function. 
The explicit form of $\delta f_{i}^{\eta}$ mentioned above depends on various ansatz (Heiselberg and Pethick, 1993; Heiselberg, 1994; Arnold et al., 2000; Dusling et al., 2010),

$$
\delta f_{i}^{\eta}=\chi(k) \frac{f_{k}^{0}\left(1 \pm f_{k}^{0}\right)}{T} \hat{k}_{i} \hat{k}_{j} \nabla_{i} u_{j}
$$

Usually, $\chi(k)$ can be determined from various microscopic theories as discussed in (Dusling et al., 2010). For the current study we are interested in a boost invariant expansion without transverse flow. In this scenario the viscous correction to the distribution function can be incorporated in the following way (Teaney, 2003; Bhatt et al., 2010; Das et al., 2013),

$$
\delta f_{i}^{\eta}(k)=f_{i}^{0}\left(1 \pm f_{i}^{0}\right) \Phi_{i}(k)
$$

where,

$$
\Phi_{i}(k)=\frac{1}{2 T^{3} \tau} \frac{\eta}{s}\left(\frac{k^{2}}{3}-k_{z}^{2}\right) .
$$

The above viscous correction holds true only in the local rest frame of the fluid and it consists of the first order correction in the expansion of shear part of the stress tensor. The flow is along $z$ axis and $\tau$ is the thermalization time of the quark-gluon plasma (QGP). It can be seen from the above expression that only in presence of momentum anisotropy the non-equilibrium part of the distribution function becomes operative.

In a medium with non-zero shear flow the expression for damping rate then becomes,

$$
\gamma(p)=\frac{1}{2 E_{p}} \int_{p^{\prime}, k, k^{\prime}} \sum_{i=1,2} \alpha_{i}(2 \pi)^{4} \delta^{4}\left(P+K-P^{\prime}-K^{\prime}\right) \frac{1}{2} \sum_{\text {spin }}|\mathcal{M}|^{2}=\gamma^{0}+\gamma^{\eta},
$$

where, $\int_{k}$ is shorthand for $\int d^{3} k /(2 \pi)^{3} 2 E_{k}$.

In the last equation $\alpha_{i}$ 's contain the information of the viscous corrected phase-space factor. $\alpha_{1}$ consists of the equilibrium part of the distribution functions. The heavy quark damping rate $\left(\gamma^{0}\right)$, where all the bath particles are in thermal equilibrium can be obtained from $\alpha_{1}$ (Thoma and Gyulassy, 1991; Thoma, 1995; Blaizot and Iancu, 1996; Blaizot and Iancu, 1997),

$$
\alpha_{1}=f_{k}^{0}\left(1 \pm f_{k^{\prime}}^{0}\right)
$$

$\gamma^{\eta}$, on the other hand, is the viscous corrected damping rate and can be derived from $\alpha_{2}$ (Sarkar and DuttMazumder, 2013),

$$
\alpha_{2} \simeq\left[\Phi_{k} f_{k}^{0}\left(1 \pm f_{k}^{0}\right) \pm \Phi_{k} f_{k^{\prime}}^{0} f_{k}^{0} \pm \Phi_{k^{\prime}} f_{k^{\prime}}^{0} f_{k}^{0}\right]
$$



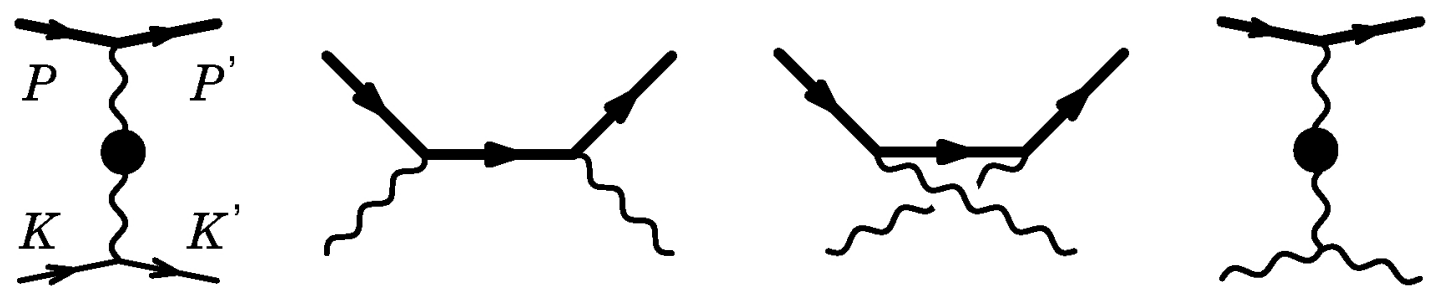

Fig. 1: Amplitudes for heavy quark $2 \rightarrow 2$ scattering in a QCD plasma

To arrive at the above expression we have neglected terms beyond $\mathcal{O}(\eta / s)$ and $\mathcal{O}\left(f_{i}^{2}\right)$. To proceed further in the evaluation of damping rate, we require the information of interaction. For Q-q scattering process in the $t$ channel, the matrix element is given by (Peigne and Peshier, 2008a; Peigne and Peshier, 2008b),

$$
\frac{1}{2} \sum_{\text {spin }}|\mathcal{M}|_{Q q}^{2} \propto \frac{\tilde{s}^{2}}{t^{2}}
$$

where, $\tilde{s}=s-m_{Q}^{2}, s$ and $t$ are the usual Mandalstam variables.

At first, the case of hard gluon exchange has been considered where the medium effects on the propagator can be ignored. In this case one encounters both logarithmic and algebraic infrared divergences in $\gamma_{Q q}^{\eta}$ of the form $\gamma_{Q q}^{\eta}(p) \propto \int d q / q$ and $\int d q / q^{3}$ unlike non-viscous medium. Usually, to handle these divergences one incorporates the effects of plasma screening. The method of inclusion of the effects of screening was first developed in (Braaten and Pisarski, 1990; Braaten and Yuan 1991). This involves introduction of an arbitrary momentum cut-off scale $q^{*}\left(g T \ll q^{\star} \ll T\right)$ to divide the region of hard momentum transfer $\left(q \gg q^{\star}\right)$ from the soft region $\left(q \ll q^{\star}\right)$. The calculation of hard momentum involves tree-level scattering diagrams whereas Hard Thermal Loop (HTL) resummed propagator is necessary for the soft momentum transfer. Since the external quark momentum is hard, inclusion of effective quark-gluon vertex is not required in the present context. Resummed quark-gluon vertex would contribute at higher order in coupling constant. The matrix amplitude with HTL resummed gluon propagator in the large wavelength limit $q \ll T$ reduces to,

$$
\frac{1}{2} \sum_{\text {spin }}|\mathcal{M}|_{Q q}^{2}=32 g^{4} E_{p}^{2} k^{2}\left[\frac{1}{\left(q^{2}+m_{D}^{2}\right)^{2}}+\frac{\left(v_{p}^{2}-\frac{\omega^{2}}{q^{2}}\right) q^{2} \cos ^{2} \phi}{\left(1-\frac{\omega^{2}}{q^{2}}\right)\left(q^{6}+\frac{\pi^{2} \omega^{2} m_{D}^{4}}{16}\right)}\right],
$$

where, $m_{D} \sim g T$ is the Debye mass. Evaluating $\gamma_{Q q}^{\eta}$ with both the hard and the soft contributions and 
restricting ourselves only to the leading logarithmic $t$ channel contribution we obtain,

$$
\begin{aligned}
\gamma_{Q q, l}^{\eta}(p) & \simeq\left(\frac{\eta}{s}\right) \mathcal{C}_{1}\left[f_{1}\left(v_{p}\right)+f_{2}\left(v_{p}\right)\right]\left(-\frac{1}{2}+\log \left|\frac{q_{\max }}{m_{D}}\right|\right) \\
& +\left(\frac{\eta}{s}\right) \mathcal{C}_{1} T^{2} f_{3}\left(v_{p}\right)\left(\frac{1}{m_{D}^{2}}-\frac{1}{q_{\max }^{2}}\right) \\
\gamma_{Q, t}^{\eta}(p) & \simeq\left(\frac{\eta}{s}\right) \mathcal{C}_{2}\left[f_{4} \int_{0}^{q^{*}} \frac{d q}{q}\right. \\
& \left.+2 \pi T^{2} m_{D}^{2} f_{5}\left(-\frac{16 v_{p}^{5}}{225}-\frac{4 v_{p}^{5}}{15} \ln \left|\frac{2 q_{\max }}{m_{D} \sqrt{\pi v_{p}}}\right|\right)\right]
\end{aligned}
$$

where $\left.\gamma\right|_{Q q, l} ^{\eta}$ and $\left.\gamma\right|_{Q q, t} ^{\eta}$ denote the longitudinal and the transverse contributions to the damping rate $\left(\left.\gamma\right|_{Q q} ^{\eta}=\right.$ $\left.\left.\gamma\right|_{Q q, l} ^{\eta}+\left.\gamma\right|_{Q q, t} ^{\eta}\right)$. The functions and constants mentioned in the above expression of the damping rate have the following forms,

$$
\begin{aligned}
\mathcal{C}_{1} & =\frac{g^{4} A_{q}}{4(2 \pi)^{3} \tau v_{p}}, f_{1}\left(v_{p}\right)=\pi^{2}\left(-\frac{v_{p}}{4}-\frac{v_{p}^{3}}{18}+\frac{v_{p}^{5}}{12}\right) \\
f_{2}\left(v_{p}\right) & =\zeta(3)\left(\frac{3 v_{p}}{2}+v_{p}^{3}-\frac{9 v_{p}^{5}}{10}\right) \\
f_{3}\left(v_{p}\right) & =\left(\frac{v_{p}}{3}-\frac{v_{p}^{3}}{3}\right)\left(\frac{7 \pi^{4}}{10}-45 \zeta(5)\right) \\
\mathcal{C}_{2} & =\frac{A_{q} g^{4} \pi T^{2}}{16(2 \pi)^{3} \tau v_{p} m_{D}^{2}}, f_{4}=\left(\frac{7 \pi^{4}}{10}-45 \zeta(5)\right) \\
f_{5} & =\left(\frac{\pi^{2}}{3}-3 \zeta(3)\right) .
\end{aligned}
$$

$q_{\max }$ in Eq. (13), can be approximated as $\sim \sqrt{E_{p} T}$ from the kinematics (Sarkar and Dutt-Mazumder, 2013) and $A_{q}=2 n_{f} / 3$ ( $n_{f}$ is the number of flavor).

$t$ channel contribution of quark-gluon (Q-g) scatterings can easily be extracted from Eq. (13). Eq. (13) excluding $A_{q}$ has to be multiplied with $A_{g}$, the color factor $A_{g}=\left(N_{c}^{2}-1\right) / 2=4\left(N_{c}\right.$ is the number of color). The total contribution of $t$ channel to the $\gamma$ is then given by $\left.\gamma\right|_{Q} ^{\eta}=-\left.\left(4+2 n_{f} / 3\right) \gamma\right|_{Q q} ^{\eta}$. In this context it would be relevant to recall the expression for heavy quark damping rate in ideal QCD plasma (Thoma and Gyulassy, 1991),

$$
\gamma(p)_{Q q}^{0}=\frac{g^{4} A_{b} T^{3}}{96 \pi}\left(\frac{1}{m_{D}^{2}}-\frac{1}{q_{\max }^{2}}\right)+\frac{g^{4} A_{b} T^{3} v_{p}}{48 \pi m_{D}^{2}} \int_{0}^{q^{*}} \frac{d q}{q},
$$


where, $A_{b}=A_{q}+A_{g}$. We now evaluate the contribution of Q-g scatterings to the viscous heavy quark damping rate through $s$ and $u$ channels. The explicit derivation can be found in (Sarkar and Dutt-Mazumder, 2013), here, we quote only the final expression,

$$
\begin{aligned}
\gamma_{Q g}^{\eta}(p) & \simeq\left(\frac{\eta}{s}\right) \mathcal{C}_{3}\left[\frac{2 \pi^{4} T^{4}}{135}-\frac{T^{3} m_{q}^{2}}{3 E_{p}}\left(2 \zeta(3) \ln \left|\frac{4 E_{p} T}{m_{q}^{2}}\right|+3 \zeta(3)\right.\right. \\
& \left.\left.-2 \gamma \zeta(3)+2 \zeta^{\prime}(3)\right)\right] .
\end{aligned}
$$

$\mathcal{C}_{3}=A_{f} g^{4} /\left(32 T^{3} \tau \pi^{3} E_{p}\right)$. The leading term of Q-g scattering rate (both in $s$ and $u$ channels) in ideal plasma is given by (Peigne and Peshier, 2008b),

$$
\begin{aligned}
\gamma(p)_{Q g}^{0} & =\frac{1}{16 \pi E_{p}} \int \frac{d^{3} k f_{k}}{(2 \pi)^{3} 2 k} \int d t \frac{|\mathcal{M}|^{2}}{s-m_{q}^{2}} \\
& =\frac{g^{4} T^{2} A_{f}}{48 \pi E_{p}}\left[\ln \left|\frac{4 E_{p} T}{m_{q}^{2}}\right|+\mathcal{O}(1)\right] .
\end{aligned}
$$

The final expression of heavy quark damping rate in viscous plasma can be obtained by adding Eqs. (13) and (16) along with the ideal contributions Eqs. (15) and (17) (Sarkar and Dutt-Mazumder, 2013),

$$
\begin{aligned}
\gamma(p)_{Q} & =\gamma(p)_{Q q}^{0}+\gamma(p)_{Q g}^{0} \\
& +\left(\frac{\eta}{s}\right) \mathcal{C}_{1}^{\prime}\left[f_{1}\left(v_{p}\right)+f_{2}\left(v_{p}\right)\right]\left(-\frac{1}{2}+\log \left|\frac{q_{\text {max }}}{m_{D}}\right|\right) \\
& +\left(\frac{\eta}{s}\right) \mathcal{C}_{1}^{\prime} T^{2} f_{3}\left(v_{p}\right)\left(\frac{1}{m_{D}^{2}}-\frac{1}{q_{\text {max }}^{2}}\right) \\
& +\left(\frac{\eta}{s}\right) \mathcal{C}_{2}^{\prime}\left[f_{4} \int_{0}^{q^{*}} \frac{d q}{q}+2 \pi T^{2} m_{D}^{2} f_{5}\left(-\frac{16 v_{p}^{5}}{225}-\frac{4 v_{p}^{5}}{15} \ln \left|\frac{2 q_{\max }}{m_{D} \sqrt{\pi v_{p}}}\right|\right)\right] \\
& +\left(\frac{\eta}{s}\right) \mathcal{C}_{3}\left[\frac{2 \pi^{4} T^{4}}{135}-\frac{T^{3} m_{q}^{2}}{3 E_{p}}\left(2 \zeta(3) \ln \left|\frac{4 E_{p} T}{m_{q}^{2}}\right|+3 \zeta(3)\right.\right. \\
& \left.\left.-2 \gamma \zeta(3)+2 \zeta^{\prime}(3)\right)\right]
\end{aligned}
$$

where, $\mathcal{C}_{1}^{\prime}=\frac{g^{4} A_{b}}{4(2 \pi)^{3} \tau v_{p}}$ and $\mathcal{C}_{2}^{\prime}=\frac{A_{b} g^{4} \pi T^{2}}{16(2 \pi)^{3} \tau v_{p} m_{D}^{2}}$.

It is evident from the above expression that divergence of the form $\int d q / q$ has been removed with the plasma effects. But logarithmic divergence which was algebraic before screening, still remains even after HTL resummation. This is reminiscent of what happens in case of the quasiparticle damping rate in ideal plasma which requires further non-perturbative treatment as discussed in (Blaizot and Iancu, 1996; Blaizot and Iancu, 1997; Boyanovsky et al., 1998). 


\section{Summary and Conclusion}

To summarize, in the present work we have calculated heavy quark damping rate in a medium where all the bath particles are affected by the shear flow of the medium. It has been shown in the text, that effect of flow of the medium enters into the calculation via the viscous corrected distribution function in the phase-space factor which in turn modifies the result. The calculation has been restricted only to the leading order contributions in $\eta / s$ by dropping all the next to leading order terms. The collision integral can be further simplified by neglecting terms beyond quadratic order in distribution functions. Furthermore, for the boson exchange we have retained only the soft frequencies following the standard formalism in non-viscous plasma. Effectively, these approximations allow us to present closed form analytical results. It is to be mentioned here that we have considered the fact that the flow has only longitudinal gradient and there is no transverse expansion. One of the interesting findings of the current study has been the infrared behavior of the transverse damping rate due to the non-existence of screening for the static gluons. To remove this divergence which remains even after using finite temperature HTL propagator one may perform further resummation by using Bloch-Nordsieck propagator or renormalization group method as already suggested in the text.

\section{Acknowledgement}

I am thankful to Late Prof. A K Dutt-Mazumder who first introduced me to this topic and fruitful discussions with him motivated me to initiate the present work.

\section{References}

1. Arnold PB, Moore GD and Yaffe LG (2000) Transport coefficients in high temperature gauge theories (I): leading-log results in JHEP 0011 001-047

2. Bhatt JR, Mishra H and Sreekanth V (2010) Thermal photons in QGP and non-ideal effects in JHEP 1011106

3. Blaizot JP and Iancu E (1996) Lifetime of Quasiparticles in Hot QED Plasmas in Phys Rev Lett 76 3080-3083

4. Blaizot JP and Iancu E (1997) Lifetimes of quasiparticles and collective excitations in hot QED plasmas in Phys Rev D55 973-996

5. Boyanovsky D, de Vega HJ, Holman R, Kumar SP and Pisarski RD (1998) Real-time relaxation and kinetics in hot scalar QED: Landau damping in Phys Rev D58 125009

6. Braaten E and Thoma MH (1991) Energy loss of a heavy quark in the quark-gluon plasma in Phys Rev D44 R2625-R2630

7. Braaten E and Pisarski RD (1990) Soft amplitudes in hot gauge theories: A general analysis in Nucl Phys B337 $569-634$

8. Braaten E and Yuan TC (1991) Calculation of screening in a hot plasma in Phys Rev Lett 66 2183-2186 
9. Das SK, Chandra V and Alam J-e (2013) Heavy-quark transport coefficients in a hot viscous quark-gluon plasma medium in $J$ Phys $\mathbf{G 4 1} 015102$

10. Dusling K, Moore GD and Teaney D (2010) Radiative energy loss and $v_{2}$ spectra for viscous hydrodynamics in Phys Rev C81 034907

11. Dusling K and Lin S (2008) Dilepton production from a viscous QGP in Nucl Phys A809 246-258

12. Dusling K (2010) Photons as a viscometer of heavy ion collisions in Nucl Phys A839 70-77

13. Dutt-Mazumder AK, Alam J-e, Roy P and Sinha B (2005) Stopping power of hot QCD plasma in Phys Rev D71 094016

14. Heiselberg H and Pethick CJ (1993) Transport and relaxation in degenerate quark plasmas in Phys Rev D48 2916-2928

15. Heiselberg H (1994) Viscosities of quark-gluon plasmas in Phys Rev D49 4739-4750

16. Moore GD and Teaney D (2005) How much do heavy quarks thermalize in a heavy ion collision? in Phys Rev C71 064904

17. Peigne S and Peshier A (2008a) Collisional energy loss of a fast muon in a hot QED plasma in Phys Rev D77 014015

18. Peigne S and Peshier A (2008b) Collisional energy loss of a fast heavy quark in a quark-gluon plasma in Phys Rev D77 114017

19. Sarkar S and Dutt-Mazumder AK (2013) Heavy quark damping rate in hot viscous QCD plasma in Phys Rev D88 054006

20. Sarkar S (2014) Modification of heavy quark energy loss due to shear flow in hot QCD plasma in arXiv : 1403.1128

21. Svetitsky B (1988) Diffusion of charmed quarks in the quark-gluon plasma in Phys Rev D37 24840-2491

22. Teaney D (2003) The Effects of viscosity on spectra, elliptic flow, and HBT radii in Phys Rev $\mathbf{C 6 8} 034913$

23. Thoma MH and Gyulassy M (1991) Quark damping and energy loss in the high temperature QCD in Nucl Phys B351 491-506

24. Thoma MH (1995) Damping rate of a hard photon in a relativistic plasma in Phys Rev D51 862-865. 